Short communication

\title{
ANTIMICROBIAL RESISTANCE IN ESCHERICHIA COLI ISOLATED FROM DIFFERENT PARTS OF THE DIGESTIVE TRACT OF SHEEP
}

\author{
E. A. AFSHARI SAFAVI ${ }^{1} \&$ Y. SHAHBAZI ${ }^{2}$ \\ ${ }^{1}$ Department of Clinical Sciences; ${ }^{2}$ Department of Food Hygiene and Quality \\ Control, School of Veterinary Medicine, Razi University, Kermanshah, Iran
}

\section{Summary}

Afshari Safavi, E. A. \& Y. Shahbazi, 2017. Antimicrobial resistance in Escherichia coli isolated from different parts of the digestive tract of sheep. Bulg. J. Vet. Med., 20, No 3, 271-275.

In order to evaluate differences in resistance patterns of Escherichia coli isolated from different parts of sheep digestive tract, the intestinal tracts of 24 sheep were sampled at various locations (duodenum, jejunum, caecum, colon and rectum) after slaughter. Samples were cultured on MacConkey agar and obtained colonies were confirmed as E. coli based on the biochemical tests results. Isolates were tested for antimicrobial agent susceptibility to 10 antibiotics (colistin, gentamicin, oxytetracycline, trimethoprim-sulfamethoxazole, amoxicillin-clavulanic acid, enrofloxacin, ampicillin, cephotaxime, neomycin and florfenicol), using disc diffusion method. The tested E. coli resistant to colistin, ampicillin and amoxicillin-clavulanic acid were isolated more frequently from large intestine (rectum) than from small intestine (duodenum) $(\mathrm{P}<0.05)$. In conclusion, antimicrobial resistance pattern of generic $E$. coli inhabiting the intestinal tract of sheep depends on sampling location, which should be considered in interpreting the results of antimicrobial resistance tests of $E$. coli isolated from the faecal samples and generalising results to bacteria colonised in other parts of the digestive tract.

Key words: antimicrobial resistance, Escherichia coli, different parts of intestinal tract, sheep

The use of antibiotics in animals for treating disease, preventing disease and improving feed efficiency have resulted in antimicrobial resistance in animal microbiomes (Allen, 2014). In some instances, antibiotic resistant bacteria could be possibly transfered from animals to man and this poses a concern in human medicine
(Marshall, 2011). Generic Escherichia coli are frequently used as indicator bacteria to monitor trends in antimicrobial resistance patterns because they are prevalent in animal and human gut flora, cultured easily and inexpensively and provides information on resistance in a population (World Health Organization, 2000; 
European Food Safety Authority, 2012). Because of the ease of obtaining, faecal sample is typically used to study antibiotic resistance pattern of bacteria of digestive tract and is commonly used to monitor the antimicrobial resistance in national surveys. For example, European Antimicrobial Susceptibility Surveillance in Animals (EASSA) examines the antimicrobial susceptibility of some bacteria including E. coli in healthy food-producing animals (beef cattle, slaughter pigs and broiler chickens) sampled at abattoir facilities (faecal or caecal samples) throughout Europe as monitoring project (Moyaert et al., 2014). However in many species including sheep, it is still unclear if the antimicrobial resistance pattern of $E$. coli isolated from faecal sample is similar to the ones isolated from upper parts of intestinal tract or not. Therefore, the main objective of this study was to evaluate differences in antimicrobial resistance pattern of Escherichia coli colonised in different parts of the digestive tract of sheep. This information is important for adjusting sampling protocols for $E$. coli as an indicator bacterium and to keep away from biased results in monitoring programs.

Samples were collected at the abattoir of Kermanshah (west of Iran). At each visit samples were collected from every $10^{\text {th }}$ sheep after evisceration at the slaughtering line and the content of the lower intestinal tract of 24 sheep was sampled at five locations (duodenum, jejunum, caecum, colon, and rectum). In upper parts (duodenum and jejunum) where intestinal content was more liquid, sampling was performed by using a sterile swab while in contact with the intestinal mucosa after emptying the contents. In other parts of the intestines, solid content was placed inside the sterile container. All samples were transported on ice to the laboratory within an hour. The faecal samples were cultured on MacConkey agar and incubated at $37{ }^{\circ} \mathrm{C}$ for $24 \mathrm{~h}$. Suspected colonies were identified as Escherichia coli based on the biochemical tests. Antimicrobial susceptibility testing was performed on Mueller Hinton agar by disk diffusion method according to the Clinical and Laboratory Standards Institute (CLSI, 2008). The following antimicrobial agents were used: colistin (CL) $10 \mu \mathrm{g}$; gentamicin (GM) $10 \mu \mathrm{g}$; oxytetracycline (T) 30 $\mu \mathrm{g}$; trimethoprim-sulfamethoxazole (SXT) 1.25/23.75 $\mu \mathrm{g}$; amoxicillin-clavulanic acid (AMX/CA) 10/20 $\mu \mathrm{g}$; enrofloxacin (NFX) $5 \mu \mathrm{g}$; ampicillin (AM) $10 \mu \mathrm{g}$; cephotaxime (CTX) $30 \mu \mathrm{g}$; neomycin (N) 30 $\mu \mathrm{g}$; and florfenicol (FF) $30 \mu \mathrm{g}$ (Padtanteb Co., Iran). Antimicrobial susceptibility data were assessed from the diameter of the zone of inhibition of growth around the disc and interpreted in accordance with CLSI standards. Escherichia coli ATCC 25922 was used as a quality control organism.

The relationship between the location of sampling and presence of antimicrobial resistance was assessed using Chi-square analysis and Fisher's exact test for each of antimicrobials separately. Data analysis was implemented using the statistical software programme (SPSS for Windows, version 16.0) and values of $\mathrm{P}<0.05$ were considered statistically significant. Isolates with intermediate susceptibility were counted with susceptible ones because the main goal was to compare the presence and absence of antimicrobial resistance between different locations of gastrointestinal tract.

Eighty nine E. coli isolates were obtained after bacteriological culture. Multiresistance (resistance to 2 or more antimicrobials) was present in 81 out of 89 $(91.01 \%)$ isolates. The number of isolates 
Table 1. Prevalence of antimicrobial resistance of generic Escherichia coli strains isolated from different parts of intestinal tract of sheep

\begin{tabular}{lllllll}
\hline $\begin{array}{l}\text { Antimicro- } \\
\text { bial drug }\end{array}$ & \multicolumn{5}{c}{ Percentage (number)of resistant isolates } \\
\cline { 2 - 7 } & Duodenum & Jejunum & Caecum & Colon & Rectum & Total \\
\hline CL & 18.8 & 27.3 & 52.6 & 52.4 & 54.5 & 43.8 \\
& $(3 / 16)^{\mathrm{A}}$ & $(3 / 11)^{\mathrm{AB}}$ & $(10 / 19)^{\mathrm{B}}$ & $(11 / 21)^{\mathrm{B}}$ & $(12 / 22)^{\mathrm{B}}$ & $(39 / 89)$ \\
GM & 18.8 & 9.1 & 10.5 & 14.3 & 18.2 & 14.6 \\
& $(3 / 16)^{\mathrm{A}}$ & $(1 / 11)^{\mathrm{A}}$ & $(2 / 19)^{\mathrm{A}}$ & $(3 / 21)^{\mathrm{A}}$ & $(4 / 22)^{\mathrm{A}}$ & $(13 / 89)$ \\
T & 93.8 & 90.9 & 100 & 95.2 & 85.7 & 93.2 \\
& $(15 / 16)^{\mathrm{A}}$ & $(10 / 11)^{\mathrm{A}}$ & $(19 / 19)^{\mathrm{A}}$ & $(20 / 21)^{\mathrm{A}}$ & $(18 / 21)^{\mathrm{A}}$ & $(82 / 88)$ \\
STX & 25 & 45.5 & 26.3 & 19 & 22.7 & 25.8 \\
& $(4 / 16)^{\mathrm{A}}$ & $(5 / 11)^{\mathrm{A}}$ & $(5 / 19)^{\mathrm{A}}$ & $(4 / 21)^{\mathrm{A}}$ & $(5 / 22)^{\mathrm{A}}$ & $(23 / 89)$ \\
AMX/CA & 12.5 & 18.2 & 44.4 & 42.9 & 50 & 36.4 \\
& $(2 / 16)^{\mathrm{A}}$ & $(2 / 11)^{\mathrm{AB}}$ & $(8 / 18)^{\mathrm{AB}}$ & $(9 / 21)^{\mathrm{AB}}$ & $(11 / 22)^{\mathrm{B}}$ & $(32 / 88)$ \\
NFX & 25 & 27.3 & 26.3 & 9.5 & 31.8 & 23.6 \\
& $(4 / 16)^{\mathrm{A}}$ & $(3 / 11)^{\mathrm{A}}$ & $(5 / 19)^{\mathrm{A}}$ & $(2 / 21)^{\mathrm{A}}$ & $(7 / 22)^{\mathrm{A}}$ & $(21 / 89)$ \\
AM & 6.2 & 9.1 & 50 & 38.1 & 40.9 & 31.8 \\
& $(1 / 16)^{\mathrm{A}}$ & $(1 / 11)^{\mathrm{AB}}$ & $(9 / 18)^{\mathrm{CD}}$ & $(8 / 21)^{\mathrm{BCD}}$ & $(9 / 22)^{\mathrm{BCD}}$ & $(28 / 88)$ \\
CTX & 13.3 & 0 & 0 & 0 & 4.5 & 3.4 \\
& $(2 / 15)^{\mathrm{A}}$ & $(0 / 11)^{\mathrm{A}}$ & $(0 / 19)^{\mathrm{A}}$ & $(0 / 21)^{\mathrm{A}}$ & $(1 / 22)^{\mathrm{A}}$ & $(3 / 88)$ \\
N & 37.5 & 50 & 42.1 & 61.9 & 54.5 & 50 \\
& $(6 / 16)^{\mathrm{A}}$ & $(5 / 10)^{\mathrm{A}}$ & $(8 / 19)^{\mathrm{A}}$ & $(13 / 21)^{\mathrm{A}}$ & $(12 / 22)^{\mathrm{A}}$ & $(44 / 88)$ \\
FF & 26.7 & 18.2 & 26.3 & 23.8 & 18.2 & 22.7 \\
& $(4 / 15)^{\mathrm{A}}$ & $(2 / 11)^{\mathrm{A}}$ & $(5 / 19)^{\mathrm{A}}$ & $(5 / 21)^{\mathrm{A}}$ & $(4 / 22)^{\mathrm{A}}$ & $(20 / 88)$ \\
\hline
\end{tabular}

CL (colistin); GM (gentamicin); T (oxytetracycline); STX (trimethoprim-sulfamethoxazole); AMX/CA (amoxicillin-clavulanic acid); NFX (enrofloxacin); AM (ampicillin); CTX (cephotaxime); $\mathrm{N}$ (neomycin); FF (florfenicol). Cells with different letters in each row are significantly different $(\mathrm{P}<0.05)$.

obtained from the upper parts was lower than lower parts (Table 1). For colistin the percentage of resistant $E$. coli isolates was significantly higher in caecum, colon and rectum than in duodenum. For amoxicillin-clavulanic acid the percentage of resistant E. coli isolates was significantly higher in rectum than in duodenum. For ampicillin the percentage of resistant $E$. coli isolates was significantly higher in colon and rectum than in jejunum and significantly higher in the caecum than in jejunum and duodenum (Table 1).
Fewer numbers of bacteria were isolated from proximal segments of intestine which can be due to the higher acidity in these parts of intestines (Sorum \& Sunde, 2001). This study indicates that the prevalence of resistance to some of the antimicrobials in Escherichia coli colonised in different parts of digestive tract of sheep depends on the site of bacterial isolation. In some previous studies conducted in other species, similar results have been obtained. Catry et al. (2007) studied antimicrobial resistance patterns of $E$. coli 
isolated from different parts of the digestive tract of veal calves and it was shown that a lot of gentamicin, nalidixic acid and enrofloxacin-resistant $E$. coli existed in the caecum, colon and rectum than in duodenum and jejunum and that the percentage of resistance to ampicillin was significantly lower in the jejunum, compared to the other segments of the intestinal tract. Moro et al. (2000) found that percentages of resistance to ampicillin for $E$. coli isolated from the ileum of swine showed significantly higher levels of resistance than isolates from caecum, colon or rectum and percentages of resistance to tetracycline were significantly higher for isolates from ileum and caecum than form colon and rectum. It has been previously shown that when sows are stressed, resistant bacteria from the upper digestive tract flow to the lower tract (Moro et al., 2000).

Therefore, the higher antimicrobial resistance against some of the antimicrobial agents in $E$. coli isolated from the faeces than E. coli isolated from the content of the upper segments in the present study could be due to the stress imposed on the animals before slaughter, especially during transportation to the abattoir. Based on the results of the present study, the antimicrobial resistance pattern of $E$. coli isolated from faeces (here rectum) can be different from isolates inhabiting other parts of sheep digestive tract. Therefore, we should be cautious in generalising the obtained results of faecal antimicrobial resistance patterns to the whole E. coli population of digestive tract of sheep. Especially these considerations should be taken into account in adjusting sampling protocols for indicator bacteria of animal origin in antimicrobial resistance monitoring programs.

Further investigations are necessary to assess the possible mechanisms involved in colonising of antibiotic-resistant Escherichia coli in different parts of sheep digestive tract.

\section{REFERENCES}

Allen, H. K., 2014. Antibiotic resistance gene discovery in food-producing animals. Current Opinion in Microbiology, 19, 25-29.

Catry, B., J. Dewulf, T. Goffin, A. Decostere, F. Haesebrouck \& A. De Kruif, 2007. Antimicrobial resistance patterns of $E s$ cherichia coli through the digestive tract of veal calves. Microbial Drug Resistance, 13, 147-150.

Clinical and Laboratory Standards Institute, 2008. Performance standards for antimicrobial disk and dilution susceptibility tests for bacteria isolated from animals, $3^{\text {nd }}$ ed. CLSI document M31-A3. Clinical and Laboratory Standards Institute, Wayne, PA.

European Food Safety Authority, 2012. The European Union summary report on antimicrobial resistance in zoonotic and indicator bacteria from humans, animals and food in 2010. The European Food Safety Authority Journal, 10, 2598.

Marshall, B. M. \& S. B. Levy, 2011. Food animals and antimicrobials: Impacts on human health. Clinical Microbiology Reviews, 24, 718-733.

Moro, M. H., G. W. Beran, R. W. Griffith \& L. J. Hoffman, 2000. Effects of heat stress on the antimicrobial drug resistance of Escherichia coli of the intestinal flora of swine. Journal of Applied Microbiology, 88, 836-844.

Moyaert, H., A. D. Jong, S. Simjee \& V. Thomas, 2014. Antimicrobial resistance monitoring projects for zoonotic and indicator bacteria of animal origin: Common aspects and differences between EASSA and EFSA. Veterinary Microbiology, 171, 279-283. 
Sorum, H. \& M. Sunde, 2001. Resistance to antibiotics in the normal flora of animals. Veterinary Research, 32, 227-241.

World Health Organization, 2000. WHO global principles for the containment of antimicrobial resistance in animals intended for food. Report of a WHO consultation, 5-9 June, Geneva, Switzerland.

Paper received 29.08.2015; accepted for publication 12.11.2015

\section{Correspondence:}

Ehsan Allah Afshari Safavi

Department of Clinical Sciences, Faculty of Veterinary Medicine, Razi University,

Resalat Bvld, Kermanshah city, Iran.

tel: +98-83-38322599,

e-mail: e.afshari@razi.ac.ir 